\title{
The association of healthy lifestyle behaviors with mental health indicators among adolescents of different family affluence in Belgium
}

\author{
L. Maenhout ${ }^{1,2^{*}}$ (D) C. Peuters ${ }^{1,2}$, G. Cardon' ${ }^{1}$ S. Compernolle ${ }^{1}$, G. Crombez ${ }^{2}$ and A. DeSmet ${ }^{3,4}$
}

\begin{abstract}
Background: Healthy lifestyles may contribute to better mental health, which is particularly important in adolescence, an age at which half of all mental health problems first occur. This association may be even more relevant in adolescents of low family affluence, who show more mental health problems, as well as more unhealthy lifestyles. This study investigated healthy lifestyle behaviors, namely sufficient sleep and physical activity, daily breakfast intake, low levels of alcohol use or smoking, in relation to mental health and symptoms of mental health problems (feelings of depression, anxiety, stress and self-esteem) among adolescents from different family affluence. Furthermore, the moderating role of family affluence was examined in those relations.
\end{abstract}

Methods: Adolescents aged 12-18y were recruited via a random sample of schools in Flanders, Belgium. A total of 1037 adolescents participated (mean age $=15.2,49.8 \%$ female). Independent samples t-tests, Mann Whitney U-tests and $x^{2}$-tests determined the differences in healthy lifestyle behaviors and mental health indicators between adolescents of low-medium and high family affluence. Regression analyses assessed the association between healthy lifestyles and mental health outcomes and the moderating role of family affluence.

Results: All healthy lifestyle behaviors were associated with at least one mental health outcome, with the exception of alcohol consumption. Adolescents from low-medium family affluence had lower levels of physical activity, less often took breakfast, had lower levels of alcohol consumption and reported lower self-esteem than adolescents from high family affluence. The results showed no moderating effect of family affluence for the association between healthy lifestyle and mental health.

Conclusion: These findings support the value of integrating healthy lifestyle behaviors in interventions for mental health promotion, for both youth of low-medium and high family affluence.

Keywords: Mental health, Adolescent, Family affluence, Healthy lifestyles, Physical activity, Breakfast, Smoking, Alcohol, Sleep

\footnotetext{
* Correspondence: Laura.Maenhout@UGent.be

${ }^{1}$ Department of Movement and Sports Sciences, Faculty of Medicine and Health Sciences, Ghent University, Ghent, Belgium

2Department of Experimental-Clinical and Health Psychology, Faculty of Psychology and Educational Sciences, Ghent University, Ghent, Belgium

Full list of author information is available at the end of the article
}

(c) The Author(s). 2020 Open Access This article is licensed under a Creative Commons Attribution 4.0 International License, which permits use, sharing, adaptation, distribution and reproduction in any medium or format, as long as you give appropriate credit to the original author(s) and the source, provide a link to the Creative Commons licence, and indicate if changes were made. The images or other third party material in this article are included in the article's Creative Commons licence, unless indicated otherwise in a credit line to the material. If material is not included in the article's Creative Commons licence and your intended use is not permitted by statutory regulation or exceeds the permitted use, you will need to obtain permission directly from the copyright holder. To view a copy of this licence, visit http://creativecommons.org/licenses/by/4.0/ The Creative Commons Public Domain Dedication waiver (http://creativecommons.org/publicdomain/zero/1.0/) applies to the data made available in this article, unless otherwise stated in a credit line to the data. 


\section{Background}

Youth mental health is defined by the World Health Organization as a state of well-being allowing youngsters to learn and acquire education, have a positive sense of identity, manage their thoughts and emotions, have a fulfilling social life and full participation in society [1]. Mental health thus exceeds the absence of mental disorders or disabilities [1]. Instead, it is a dynamic ability to find a balance between all aspects of life [2]. Poor mental health is a severe public health concern, particularly in adolescents. Half of all mental health problems start by the age of 14, making adolescence a crucial period for mental health promotion [3]. Worldwide, it is estimated that $10-20 \%$ of adolescents experience mental health problems [4]. In Belgium, 80.9\% of adolescents between 2 and 18 years of age do not report an emotional problem (defined as a mental health problem with clear, excessive and persistent signs of depression, anxiety, panic, phobia or other emotional problems), $9 \%$ is considered a borderline case and $10 \%$ has a suspected pathology [5]. Evidence-based treatments for mental health problems, such as psychotherapy and pharmacotherapy, form a cornerstone of care available for those suffering from mental health problems. Such treatments, however, also face challenges: only small effects of treatment were found for a large group of patients $[6,7]$, young people are often reluctant to seek professional help [8], treatment comes at a high price for individuals and health care systems, and such treatments may have a range of undesirable side-effects [9]. In this context, prevention of mental health problems is of utmost importance.

Mental health is influenced by many factors, including everyday behaviors that can be altered by individuals $[10,11]$. Mental health programs that support adolescents in managing their mental health by improving everyday health behaviors, are empowering, destigmatizing, and can have a large impact at population level at low cost [6]. Several modifiable risk and protective factors have been identified in adolescents: sufficient sleep and physical activity and a healthy diet were associated with better mental health outcomes, such as lower depression, anxiety, stress $[6,11-16]$ and higher selfesteem [14, 15, 17-20]; whereas high alcohol consumption and smoking were associated with less beneficial mental health outcomes, such as higher psychological distress, depression, anxiety, stress [6, 21-23], and lower self-esteem [24, 25]. There is room for improvement in the area of healthy lifestyles among Flemish adolescents: half do not meet the norm of 8-h of sleep; most do not engage in sufficient physical activity; and around half do not take breakfast daily [26-30]. Although the rate has slightly decreased, a substantial number of adolescents engages in drinking (34.4-36\%) and smoking (2.4-4.3\%) $[31,32]$. Increasing healthy behaviors (sufficient sleep and physical activity, and a healthy diet) and reducing these unhealthy behaviors (alcohol consumption and smoking) can increase adolescents' resilience and mental well-being.

In most countries, adolescent health outcomes are associated with socioeconomic status (SES) [33-37]. Adolescents of low SES thus form a particular group of interest for promoting mental health via healthy lifestyles. Both mental health and healthy lifestyles in adolescents of low SES are found to be poorer than among adolescents of high SES. A systematic review [38], which included studies that measured SES in various ways (i.e., through parental occupation, income or education), showed that socioeconomically disadvantaged children and adolescents are two to three times more likely to develop mental health problems [38-40] than their peers from socioeconomically advantaged families. In addition, adolescents from low SES reported lower levels of sleep, physical activity and healthy diet, and higher levels of smoking than teens from high SES [28-30, 32, 34, 41-50]. Research on how adolescents' alcohol consumption differed as a function of SES was inconsistent [31, 47, 49, 51-53]. It can be expected that unhealthy lifestyles have an even stronger association with mental health in adolescents of lower SES than of higher SES, since both healthy lifestyles and mental health are found to be poorer in this population. To our knowledge, only two studies so far have addressed the associations between healthy lifestyle behaviors and mental health in adolescents of low SES. These studies showed positive associations, but focused on only one health behavior (i.e., physical activity or sleep) in relation to psychological distress $[43,54]$. The present study combines different health behaviors and includes positive well-being in defining mental health. Moreover, SES is measured using the Family Affluence Scale [55], as this can be easily answered by adolescents themselves. Different ways exist to measure 'SES'. Many researchers refer to the general term 'SES', but use specific indicators (i.e. income, occupation, education, etc.). This makes it difficult to compare study results. When referring to our specific SES-indicator (i.e. FAS), we have therefore consistently used 'FAS' to be specific and clear. However, when we are referring to other studies or comparing our results with previous studies, we will use the general term 'SES', since other researchers have used different indicators to refer to SES. The aim of the current study is to investigate: 1) associations between healthy lifestyles, namely sufficient sleep duration and physical activity, daily breakfast intake, low levels of alcohol use or smoking, and mental health (research question RQ1); 2) the level of healthy lifestyle behaviors and mental health among adolescents from different family affluence (RQ2); and 3) the moderating role of family affluence in the relation between healthy lifestyles and mental health (RQ3). It is hypothesized that 
healthier levels of lifestyle behaviors are associated with better mental health ( $\mathrm{H} 1)$; that adolescents from lower family affluence report lower levels of healthy lifestyles and poorer mental health than adolescents of higher family affluence (H2); and we expect to find that family affluence plays a moderating role in the relation between the investigated healthy lifestyle behaviors and mental health outcomes (H3). Our results may indicate which lifestyle behaviors are associated with adolescent mental health, and may encourage health professionals in designing programs to lower the risk for mental health problems among adolescents. In addition, these results can shed a light on health promotion strategies particularly effective in lower family affluent groups in youth.

\section{Methods}

\section{Participants and data-collection}

We selected a random sample of schools $(n=26)$ from a government database of secondary schools in Flanders, a region in Belgium consisting of around 6 million inhabitants. All included schools were state-funded, as the vast majority of schools in Belgium are state funded. Eight schools (31\%) agreed to collaborate in the study. The main reason for not participating was no time to set-up the survey at the school within the time frame of the study. The study took place between November 2014 and May 2015. Within each school, classes were randomly selected. We aimed to collect data among grades 7 to 12 for each school (aged 12-18). Data collection took place at school, during one class hour. The anonymous paper-and-pencil survey was administered by the researchers, who explained at the start of the survey that students were under no obligation to participate and could withdraw at any time. Students were assured that their responses would be confidential and that no information would be shared with teachers, parents, or fellow students. Five students declined to participate, none of the parents declined consent. The study received approval from the Ethics Committee of the Ghent University Hospital (2012/307, B670201214183). Adolescents provided written informed consent, parents provided passive informed consent. Parents were informed about the study through the school and received a telephone number and e-mail address from the researchers, via which they could notify that they did not want their child to participate. They were informed that when they did not contact the researchers, they agreed with participation of their child.

\section{Measures}

\section{General socio-demographic information}

Items were selected from the Health Behavior in Schoolaged Children (HBSC) 2009/10 questionnaire, a crossnational survey supported by the World Health
Organization [56]. Socio-demographic variables included gender, age, type of education (general academic, technical or vocational track), country of birth, family living situation, self-reported weight and height (used to calculate Body Mass Index, BMI). Age, gender and BMI were taken into account as covariates in this study, as gender, age and BMI differences have been associated with adolescents' health behaviors and (mental) health outcomes [57]. For example, girls are less likely to have breakfast every weekday and boys, in general, report early and weekly smoking more often. In addition, girls are more likely to describe lower life satisfaction in comparison to boys [57]. Furthermore, a negative drop in healthy behaviors is seen with increasing age. For example, 11-year-olds are more likely to meet the physical activity guidelines of at least $60 \mathrm{~min}$ of moderate to vigorous physical activity daily than 15-yearolds in almost all countries and regions [57]. BMI has also been reported as inversely associated with global selfesteem in adolescents [58].

Family affluence This part of the HBSC questionnaire also consists of the validated adolescent self-report 'Family Affluence Scale' (FAS), to identify family material wealth and socio-economic status (SES) of children and adolescents [55, 59-61]. The FAS is used as an indicator of SES. It has widely been used to explore and explain socioeconomic inequalities in a wide range of health indicators in the HBSC study over the last 20 years [60]. FAS is validated against other measures of SES and macro-economic indicators (e.g. Gross Domestic Product (GDP)) in 35 countries $[55,60]$. The FAS was developed to overcome the problem of inaccurate perceptions and missing data among children and adolescents of their family's finances, especially among lower socioeconomic groups which could thus lead to an underestimation of socioeconomic inequalities [36, 60]. It was proposed as a less intrusive, more comprehensible approach to identify the family's socioeconomic status [62] than inquiring about parents' educational, occupation or income levels $[55,63]$. It is indicated that in contrast to for example parental occupation, the proportion of missing data on FAS items is low [60]. The FAS II consists of four items: number of cars, own bedroom, computers owned and number of holidays per year [55, 62]. A composite FAS score (ranging from 0 to 9 ) is calculated for each adolescent based on his or her responses to these four items. The following, international, cut-off points were used: score of $0,1,2$ classified as low affluence; score of 3, 4, 5 as medium affluence; and a score of 6,7 , 8, 9 classified as high affluence [55].

\section{Healthy lifestyles}

Items to assess healthy lifestyles, except for sleep duration, were also taken from the HBSC survey. Several 
health-related lifestyle behaviors among adolescents are interrelated. Based on Principal Component Analyses on these data reported elsewhere [12], healthy lifestyles were grouped into two factors: 'energy-balance related behaviors', consisting of physical activity and a healthy diet, and 'addictive behaviors and sleep duration', consisting of alcohol consumption, smoking and perceived sleep duration. These factors will be used to discuss the results, individual behaviors are however retained in the analyses.

\section{Energy-balance related behaviors}

Physical activity was measured by the number of days they achieved $\geq 60 \mathrm{~min}$ of moderate to vigorous physical activity, and was defined in the questionnaire as: "bodily movements that make your heart beat faster and make you feel out of breath at some moments". A healthy diet was measured by assessing the number of days per week adolescents had have breakfast. Eating a regular, healthy breakfast contributes to the daily recommended intake of essential nutrients [64, 65]. Moreover, a daily breakfast may be used to identify adolescents at risk for unhealthy lifestyle behaviors. For example, daily breakfast intake is associated with both daily fruit and vegetable consumption, and there is an inverse relationship between daily breakfast intake and daily soft drink consumption [50].

\section{Addictive behaviors and perceived sleep duration}

Alcohol use was assessed by summing the frequency of six different types of alcohol consumption: beer, wine, spirits/liquor, alcopops and any other drink that contains alcohol (0-never; 4-daily. Range of summed score 0-24). Three questions were asked on tobacco use, based on the Flemish version of the HBSC 2009/10 questionnaire: 1) have you ever smoked tobacco?; 2) how often do you smoke currently?; 3) how many cigarettes per day have you smoked on average over the last 30 days?. These questions were combined and recoded to form one indicator of tobacco use frequency, namely: 0 'never smoked'; 1 'I have smoked but do not smoke now'; 2 'I smoke now, but I am not a daily smoker'; 3 'I smoke daily, but I am low dose smoker'; and 4 'I smoke daily and am a high dose smoker'. To decide on low dose and high dose among daily smokers, the median among the group of daily smokers was used as a cut-off for tobacco use frequency. The median showed around half of daily smokers smoked fewer than 11 cigarettes per day (=low dose), and half smoked 11 cigarettes or more per day (= high dose). Self-reported smoking is a reliable indicator of smoking status [66]. All questions from smoking were derived from the HBSC study where items have been decided by an international expert team and have been used in numerous studies [67-69]. Specifically, for the three questions on smoking ICC-values of $0.75,0.50$ and 0.85 have been reported [70]. To calculate sleep duration (number of hours slept per night), adolescents were asked to report at what time they usually go to bed and get up. Self-reported duration of sleep is strongly correlated with sleep duration measured by accelerometers for weeknights and moderately correlated with sleep duration for weekend nights [71].

\section{Mental health}

Mental health was measured through feelings of depression, anxiety and stress and self-esteem. Feelings of depression, anxiety and stress were measured with the Depression Anxiety Stress Scales (DASS-21) which has good psychometric properties to measure adolescent mental health outcomes [72, 73]. It consists of seven items per subscale [73]. Total scores per subscale were used as dependent variables, with high reliability for each of the subscales $\left(\alpha_{\text {depression }}=0.90 ; \alpha_{\text {anxiety }}=0.84\right.$; $\alpha_{\text {stress }}=0.87$ ). Focusing on self-esteem is considered a core element of mental health promotion and a fruitful basis for a broad-spectrum approach [74]. Positive global self-esteem was measured by a single item from the Rosenberg Self-Esteem Scale (RES), namely 'I take a positive attitude toward myself'. Global self-esteem can be measured by a single item [75] and this specific item is a main contributor to global positive self-esteem $[76,77]$.

\section{Analysis}

Variables were checked on normal distribution with the values for skewness and kurtosis. The values for skewness and kurtosis between -2 and +2 are considered acceptable in order to prove normal univariate distribution [78]. To test the significance of difference of the health behaviors and mental health indicators between lowmedium and high FAS (Table 1), independent samples ttests were used for variables with a normal distribution (i.e., physical activity, healthy diet, sleep duration, alcohol consumption and self-esteem) and the nonparametric variant, Mann-Whitney U tests were used for the variables that did not have a normal distribution (i.e., symptoms of depression, anxiety and stress). For smoking, a $X^{2}$-test was conducted. Gamma regression models were used to account for the positively skewed distribution of the dependent variables: depression, anxiety and stress [79]. The dependent variable 'self-esteem' showed a normal distribution. For this variable, multiple linear regression analysis was used. Regression analyses assessed the association between healthy lifestyles and mental health outcomes (RQ1); and the moderating role of family affluence in the relation between healthy lifestyles and mental health outcomes (RQ3). Analyses were controlled for individual background factors that significantly influenced mental health outcomes (namely, gender, age and BMI). Analyses were conducted stepwise, by first examining the influence of family affluence and 
Table 1 Sample characteristics and differences between (in) dependent variables

\begin{tabular}{|c|c|c|c|c|}
\hline & $\begin{array}{l}\text { Full sample } \\
(n=1037)\end{array}$ & $\begin{array}{l}\text { Low-med family } \\
\text { affluence }(n=179)\end{array}$ & $\begin{array}{l}\text { High family affluence } \\
(n=838)\end{array}$ & $\begin{array}{l}\text { Significance of } \\
\text { difference }\end{array}$ \\
\hline \multicolumn{5}{|l|}{ Physical activity } \\
\hline$\geq 60$ min. Physical activity (number of days/week), mean \pm SD & $3.33 \pm 2.09$ & $2.83 \pm 2.07$ & $3.40 \pm 2.08$ & $t(1,990)=-3.32^{* * a}$ \\
\hline \multicolumn{5}{|l|}{ Healthy diet } \\
\hline Breakfast (number of days/week), mean \pm SD & $5.38 \pm 2.19$ & $4.88 \pm 2.38$ & $5.50 \pm 2.13$ & $t(1,999)=-3.16^{* * a}$ \\
\hline \multicolumn{5}{|l|}{ Alcohol consumption } \\
\hline Frequency of alcohol consumption (0-24), mean \pm SD & $3.36 \pm 3.85$ & $2.82 \pm 3.60$ & $3.45 \pm 3.86$ & $t(1,994)=-1.96^{* a}$ \\
\hline \multicolumn{5}{|l|}{ Smoking } \\
\hline Percentage (\%) current smokers (daily + non-daily) & 12.4 & 14.6 & 11.9 & $x^{2}=0.95^{c}$ \\
\hline Percentage (\%) high dose daily smokers & 4.4 & 7.0 & 4.0 & $x^{2}=3.05^{\circ} \mathrm{c}$ \\
\hline \multicolumn{5}{|l|}{ Perceived sleep duration } \\
\hline Average hours of sleep/night, mean \pm SD & $7.87 \pm 1.42$ & $7.95 \pm 1.55$ & $7.85 \pm 1.39$ & $t(1,984)=0.83^{a}$ \\
\hline \multicolumn{5}{|l|}{ Mental health outcomes } \\
\hline Symptoms of depression, mean \pm SD & $6.18 \pm 8.51$ & $6.80 \pm 9.35$ & $6.09 \pm 8.34$ & $Z=-0.29^{b}$ \\
\hline Anxiety, mean $\pm S D$ & $5.44 \pm 7.12$ & $5.60 \pm 7.23$ & $5.45 \pm 7.09$ & $Z=-0.10^{b}$ \\
\hline Stress, mean $\pm S D$ & $7.93 \pm 8.11$ & $8.30 \pm 8.23$ & $7.92 \pm 8.10$ & $Z=-0.51^{b}$ \\
\hline Self-esteem, mean \pm SD & $3.71 \pm 1.09$ & $3.52 \pm 1.15$ & $3.76 \pm 1.06$ & $t(1,973)=-2.6^{* * a}$ \\
\hline
\end{tabular}

${ }^{\circ} p \leq .1 ;{ }^{*} p \leq 0.05 ;{ }^{* *} p \leq .01 ;{ }^{* * *} p \leq .001$

aindependent samples t-tests

${ }^{\mathrm{b}}$ Mann Whitney U-tests

${ }^{c}{ }^{2}$-tests

background variables, next the healthy lifestyle variables, and finally the interaction effects between healthy lifestyle variables and family affluence. Because no interaction effects were significant, the parsimonious model was constructed based on the full model of the direct effects (see Tables 2 and 3). In the first regression analyses (Table 2), BMI was not a significant predictor for any mental health outcome and therefore not included in further regression analyses (Table 3). Collinearity diagnostics were conducted examining Variance Inflation Factor (VIF) $(\leq 10)$ and tolerance $(\geq 0.1)$. VIF showed no multicollinearity among independent variables. Crosstabulations were checked for empty combinations of cells or low expected frequencies [80]. Continuous independent variables were mean centered. Moderator variables were created by multiplication of interaction variables. All analyses were conducted in SPSS 25.0.

\section{Results}

The initial sample consisted of 1062 adolescents, from which 25 were removed due to incomplete or unsatisfying answers (no variation on relevant diverging questions or nonsense answers to open-ended questions), resulting in an analyzed sample of 1037 adolescents $(49.8 \%$ female; mean age $=15.17 \mathrm{y} \pm 1.86$; mean $\mathrm{BMI}=$ $19.56 \pm 3.70)$. For 20 adolescents, no FAS score could be

Table 2 Regression analysis on the moderating role of family affluence in the relation between addictive behaviors and sleep, and mental health outcomes

\begin{tabular}{|c|c|c|c|c|}
\hline & \multicolumn{4}{|c|}{ Dependent variable: mental health outcomes } \\
\hline & Depression $^{a}$ & Anxiety $^{a}$ & Stress $^{\mathrm{a}}$ & Self-esteem ${ }^{\mathbf{b}}$ \\
\hline \multirow[t]{2}{*}{ Parsimonious model results (direct effects) } & $\begin{array}{l}B I C=4753.20 \\
C A I C=4758.20\end{array}$ & $\begin{array}{l}B / C=4721.28 \\
C A I C=4726.28\end{array}$ & $\begin{array}{l}B / C=5694.01 \\
C A / C=5699.01\end{array}$ & $F(3,938)=21.26, p<.001$, adj. $R^{2}=0.06$ \\
\hline & $\operatorname{Exp}(B),(95 \% \mathrm{Cl})$ & & & $\beta$ (B; SE) \\
\hline Gender (ref. girls) & $0.68(0.56 ; 0.82) * * *$ & $0.66(0.55 ; 0.79) * * *$ & $0.72(0.61 ; 0.84)^{* * *}$ & $0.19(0.41 ; 0.07)^{* * *}$ \\
\hline Family Affluence & Not included & Not included & Not included & $0.10(0.29 ; 0.09)^{* * *}$ \\
\hline Smoking & $1.15(1.05 ; 1.27)^{* *}$ & $1.17(1.07 ; 1.28) * * *$ & $1.16(1.07 ; 1.26)^{* * *}$ & Not included \\
\hline Sleep & $0.86(0.80 ; 0.92) * * *$ & $0.87(0.82 ; 0.93) * * *$ & $0.91(0.86 ; 0.97)^{* *}$ & $0.15(0.12 ; 0.02)^{* * *}$ \\
\hline
\end{tabular}

${ }^{\circ} p \leq .1 ; * 0 \leq 0.05 ;{ }^{* *} p \leq .01 ;{ }^{* * *} p \leq .001$

${ }^{a}$ Gamma generalized linear model

${ }^{\mathrm{b}}$ General linear model 
Table 3 Regression analysis on the moderating role of family affluence in the relation between energy-balance related behaviors and mental health outcomes

\begin{tabular}{|c|c|c|c|c|}
\hline & \multicolumn{4}{|c|}{ Dependent variable: mental health outcomes } \\
\hline & Depression $^{\mathrm{a}}$ & Anxiety $^{\mathrm{a}}$ & Stress ${ }^{a}$ & Self-esteem $^{\mathbf{b}}$ \\
\hline \multirow[t]{2}{*}{ Parsimonious model results (direct effects) } & $\begin{array}{l}B / C=4619.84 \\
C A \mid C=4625.84\end{array}$ & $\begin{array}{l}B I C=4605.30 \\
C A I C=4611.30\end{array}$ & $\begin{array}{l}B I C=5574.12 \\
C A I C=5579.12\end{array}$ & $F(3,951)=20.12, p<.001$, adj. $R^{2}=0.06$ \\
\hline & $\operatorname{Exp}(B),(95 \% \mathrm{Cl})$ & & & $\beta$ (B; SE) \\
\hline Age & $1.05(1.00 ; 1.11)^{\circ}$ & $1.04(0.99 ; 1.10)$ & $1.04(1.00 ; 1.09)^{\circ}$ & Not included \\
\hline Gender (ref. girls) & $0.69(0.57 ; 0.85)^{* * *}$ & $0.68(0.56 ; 0.81)^{* * *}$ & $0.72(0.61 ; 0.85)^{* * *}$ & $0.18(0.39 ; 0.07)^{* * *}$ \\
\hline Family Affluence & Not included & Not included & Not included & $0.08(0.22 ; 0.09)^{*}$ \\
\hline Physical activity & $0.92(0.88 ; 0.97)^{* * *}$ & $0.96(0.92 ; 1.01)^{\circ}$ & Not included & Not included \\
\hline Days of breakfast & $0.95(0.91 ; 0.99)^{*}$ & $0.93(0.89 ; 0.97)^{* * *}$ & $0.94(0.91 ; 0.98)^{* *}$ & $0.15(0.07 ; 0.02)^{* * *}$ \\
\hline
\end{tabular}

${ }^{\circ} p \leq .1 ;{ }^{*} p \leq 0.05 ;{ }^{* *} p \leq .01 ;{ }^{* * *} p \leq .001$

${ }^{a}$ Gamma generalized linear model

${ }^{\mathrm{b}}$ General linear model

calculated due to missing information. The majority of the adolescents had a high family affluence (82.4\%), which is in line with the high affluence rate in this region reported in the HBSC 2009/10 study (i.c. 72.7\%; Buijs, T., personal communication). Most adolescents were born in Belgium (94.0\%). Around two third lived with both parents (64.1\%), one third had another family situation (e.g. living with one parent or in coparenthood, living with other family members).

Results on sample characteristics can be found in Table 1 . On average the sample performed $\geq 60 \mathrm{~min}$. of moderate to vigorous physical activity on 3,3 days a week; they took breakfast on 5,38 days a week; and slept $7.87 \mathrm{~h}$ per night. Moreover, the sample had a relatively low frequency of alcohol consumption (sum score 3.36/ 24 ) and $12,4 \%$ were current smokers. Adolescents of high family affluence were physically active on significantly more days, took breakfast on more days, reported higher alcohol consumption and had higher self-esteem than adolescents of lower family affluence. There were no significant differences in smoking, sleep duration, or other mental health outcomes between adolescents of low-medium and high family affluence (RQ2).

Table 4 (full table in Appendix) shows the main effects of addictive behaviors and sleep on mental health outcomes, as well as the results of the moderating role of family affluence in the relation between these behaviors and mental health outcomes (RQ1 and 3). A lower sleep duration was significantly associated with lower mental health on all studied indicators. More smoking had a main effect on more symptoms of depression, anxiety and stress. Alcohol consumption was not significantly associated with any of the mental health outcomes. No moderating effect of family affluence was found in these relations with mental health outcomes. Family affluence was found as a significant main predictor of self-esteem.
Table 5 (full table in Appendix) shows the associations between the energy-balance related behaviors and mental health, as well as the results of the moderating role of family affluence in the relation between energy-balance related behaviors and mental health outcomes (RQ1 and 3). Daily breakfast intake was associated with higher mental health on all outcomes. Higher levels of physical activity only showed a significant main effect on one of the mental health outcomes, i.c. lower feelings of depression. There was no moderating role of family affluence in the relation between energy-balance related behaviors with any of the mental health outcomes. Family affluence, however, showed a significant association with self-esteem: youth of low-medium family affluence had lower self-esteem than youth of high family affluence.

\section{Discussion}

This study investigated healthy lifestyle behaviors and mental health among adolescents, thereby differentiating between adolescents from low to medium and high family affluence, and examining whether family affluence plays a moderating role in the relation between certain healthy lifestyles and mental health outcomes. Findings indicated that healthy lifestyle behaviors were indeed associated with better mental health outcomes, and that certain but not all healthy lifestyle behaviors and mental health outcomes were lower among adolescents of low to medium family affluence than those of high family affluence. We, however, did not find that family affluence moderated the association between healthy lifestyles and mental health outcomes. This indicated that healthy lifestyles are important in mental health among adolescents for both adolescents of low-medium and high family affluence.

The results show that all healthy lifestyle behaviors were associated with at least one mental health outcome, with the exception of alcohol consumption. Lower sleep 
duration and daily breakfast intake were significantly associated with lower mental health on all studied indicators. Higher levels of physical activity only showed a significant association with one of the mental health outcomes, i.e. lower feelings of depression. More smoking showed an association with higher levels of feelings of depression, anxiety, and stress, but not with self-esteem. This pattern of results was, however, not completely in line with our idea that all forms of healthy lifestyles would be associated with all of the mental health outcomes (lower feelings of depression, anxiety and stress and higher self-esteem). Only sufficient sleep and daily breakfast intake were related to all mental health outcomes included in this study. It may be that depending on the mental health outcome, other healthy lifestyle behaviors are important. In this sense, combining different healthy lifestyle behaviors in a mental health promotion intervention may be beneficial, as various mental health outcomes are important for a positive mental well-being of adolescents.

The results only partially support the hypothesis that adolescents from lower family affluence would engage in less healthy levels of lifestyle behaviors and experience poorer mental health outcomes than adolescents from higher family affluence. Consistent with previous studies [39, 40, 42, 47], the present study shows that adolescents from low-medium family affluence had lower levels of physical activity, less often took breakfast, had lower levels of alcohol consumption and reported lower self-esteem than adolescents from high family affluence. No significant differences between youngsters of low-medium and high family affluence were found for sleep duration, smoking, and for feelings of depression, anxiety and stress. Regarding sleep and smoking, previous studies reported that adolescents from lower family affluence had poorer sleep duration $[41,43]$ and higher levels of cigarette smoking [46-49] than adolescents from high family affluence. Our findings regarding sleep duration and cigarette smoking were therefore not consistent with previous studies. In the area of alcohol consumption, previous studies that compared adolescents of low family affluence with adolescents of high family affluence have reported inconsistent results $[47,51-53]$. Our findings show a small difference, indicating that adolescents of high family affluence on average reported higher alcohol consumption than adolescents of low-medium family affluence. A possible explanation may be that adolescents with more pocket money are able to buy more alcohol than adolescents with less pocket money [51, 81]. However, other studies also have reported weak, inverse or no links in alcohol consumption between lowmedium and high family affluence $[47,52,53]$.

Some of the differences between our findings and the literature may be due to different ways of measuring SES. Prior work already indicated that relationships between healthy lifestyles and SES may be inconsistent across SES indicators [34, 38, 49, 53]. In our study, an adolescent self-report measure, namely FAS, was used to identify SES of adolescents [55]. This in comparison with studies that measure (parental) SES through income, education or occupation [41, 43, 48, 49, 51, 52]. FAS measures only one aspect of SES, which is much more related to material wealth, income and spending patterns $[34,82]$. In line with our study, for example, Richter et al. reported a small effect of family affluence on alcohol consumption; indicating an increasing risk of higher alcohol consumption with increasing family affluence, whereas no significant association was observed for educational track [53]. Furthermore, physical activity and daily breakfast intake might be influenced more by financial resources (i.e., possibility of registering in a sports club or purchasing healthy food) than sleep or smoking. Those latter behaviors may be more strongly associated with education and occupational status than with income or material wealth [34]. Parental occupation reflects to some degree parents' educational status. Educational strategies, values, norms and model behavior of parents may be more likely to positively influence sleep or smoking [34, 82]. However, it is difficult to draw a clear line in this, as we can imagine that buying cigarettes in many countries is also expensive, and having a daily breakfast can be strongly influenced by parents' modelling behavior. Moreover, FAS associations are strong for health outcomes that are related to family culture and behavior (such as physical activity and healthy diet), but less so for some behaviors with strong peer norms (like alcohol use and smoking). Those addictive behaviors (alcohol use and smoking) might be less strongly influenced by parental socioeconomic status $[34,53]$. In this study, there was only a small significant difference in alcohol consumption between low-medium and high family affluence. Richter et al. concluded that other determinants (like for example peers or school setting) might have a larger impact on adolescent alcohol consumption than parental SES (measured with the FAS) or adolescents' own SES (measured through educational track) [53]. In general, it is important that further research explores to what extent the different indicators of SES influence adolescent healthy lifestyle behavior as this could give important insights for preventive strategies [34].

Although this study shows that healthy lifestyles are clearly significant predictors of mental health, and there are some differences in healthy lifestyles according to family affluence, we did not find any significant differences in symptoms of mental health problems (feelings of depression, anxiety and stress) between adolescents from low-medium family affluence and adolescents from high family affluence. Prior work indicates that low family affluence tends to be more strongly related with externalizing problems (e.g., attention deficit hyperactivity 
disorder, conduct disorder, antisocial behavior) than with internalizing problems (such as depression, anxiety and stress that were included here) among children and adolescents [38]. Moreover, it may well be that other unmeasured factors contribute to mental health. For example, social support from friends and spending time with friends during leisure time are strong protective factors against symptoms of depression and anxiety in adolescents [83], especially in those living in areas of low socioeconomic disadvantage [84]. To conclude, we see that adolescents with low-medium FAS report lower levels of self-esteem, but that this is not the case for symptoms of depression, anxiety and stress. Future research may identify possible underlying reasons.

This study furthermore shows no moderating effect of family affluence. This indicates that the relationships between lifestyle behaviors and mental health outcomes exist for both adolescents of low-medium and high family affluence. This would mean that mental health interventions can focus on the healthy lifestyle behaviors for adolescents of all forms of family affluence. Adolescents of low-medium family affluence may however need more or different support in reaching these healthy lifestyles and mental health outcomes, as they to some extent still report lower levels of healthy lifestyle behaviors and poorer mental health outcomes. Future research needs to explore how such interventions may be best designed.

\section{Conclusion}

Attention should continue to be paid to (mental) health inequalities between adolescents of low-medium family affluence and high family affluence. Poor mental health among adolescents of low-medium family affluence (as of other family affluence) might be reduced by improving health-related behavior. Our study concluded that all included healthy lifestyle behaviors are associated with at least one of the mental health outcomes, with the exception of alcohol consumption. Adolescents can tackle these behaviors in their daily lives to reduce their risk of mental health problems and build their resilience, and should therefore be integrated in interventions for mental health promotion. This was to our knowledge the first study to assess whether family affluence plays a moderating role in the association between these aforementioned different healthy lifestyle behaviors and mental health outcomes. No moderating effect of family affluence was found.

\section{Limitations and strengths}

This study had some limitations. First, our design is crosssectional in nature. Hence, the causal direction of these relationships cannot be determined. Intervention or longitudinal studies are needed to assess whether healthy lifestyle behaviors have an effect on mental health indicators. Second, there is a wide variety of SES-measures across studies in literature. The inconsistent use of these SES-measures complicates comparisons, explanations and interpretations. Third, the majority of our sample was highly affluent, consistent with the high affluence of the country. This may limit the generalizability of our findings to other countries with a lower national level of affluence. Fourth, the explained variance of the healthy lifestyles in relation to mental health outcomes was quite small. Even though various healthy lifestyle behaviors were analyzed there are, of course, other important (lifestyle) factors that were not included in this study (e.g., social support, (social) media influences, relation with peers, etc.) $[83,85,86]$. Mental health promotion programs may therefore consider to also include other components besides healthy lifestyles. Furthermore, interpreting adolescents' lifestyle behavior obtained from self-reports can be difficult as these may be influenced by social desirability. Nevertheless, we expect a low social desirability bias given the survey's anonymity. In line with this, also adolescents' weight and height for calculating BMI were self-reported; such results may be biased [87]. Despite the widespread use of the four items in FAS II, they may not be bias-free, especially in crossnational contexts. The FAS-items should continue to be updated to reflect material affluence of the family across countries. To reduce the burden for the adolescents, daily breakfast intake was the only indicator of healthy diet. However, a healthy diet consists also of other aspects of nutrition (e.g., fruit, vegetables, fish, etc.) [88-90]. Lastly, our study investigated alcohol consumption as one of the (un) healthy lifestyles. For some adolescents, alcohol consumption may be prohibited by their religion, and this may also impact results. We however did not question adolescents' religious or cultural restrictions regarding alcohol consumption, this would be a valuable aspect to take into account in future research. The study also had several strengths. First of all, mental health was defined using a broad concept of both positive well-being and mental health problems, in line with the WHO conceptualization of mental health. Family affluence was measured using a validated scale that provides reliable information based on adolescents' self-reports [55]. The FAS has the advantage that it can be easily answered by youth. Furthermore, the FAS makes international comparisons possible, as this scale is used in all the HBSC-studies across different countries. It is indicated that the FAS may be more ecologically valid than parental income data since it is based on the family context of consumption $[55,63]$. Our study added to the scarce research on healthy lifestyles to improve youth mental health and how this differed by youth's family affluence. Our study showed that healthy lifestyles differ between youth of low-medium and high family affluence and that these healthy lifestyles may contribute to a better mental health for all. 


\section{Appendix}

Table 4 Regression analysis on the moderating role of family affluence in the relation between addictive behaviors and sleep, and mental health outcomes

\begin{tabular}{|c|c|c|c|c|}
\hline & \multicolumn{4}{|c|}{ Dependent variable: mental health outcomes } \\
\hline & Depression $^{a}$ & Anxiety $^{a}$ & Stress $^{a}$ & Self-esteem ${ }^{b}$ \\
\hline \multirow[t]{2}{*}{$\begin{array}{l}\text { Full model results (direct effects, only } \\
\text { family affluence) }\end{array}$} & $\begin{array}{l}B I C=5007.032 \\
C A I C=5010.032\end{array}$ & $\begin{array}{l}B I C=5007.17 \\
C A I C=5010.17\end{array}$ & $\begin{array}{l}B / C=6010.34 \\
C A I C=6013.34\end{array}$ & $F(1,973)=7.57, p=0.006$, adj. $R^{2}=0.01$ \\
\hline & \multicolumn{3}{|l|}{$\operatorname{Exp}(B),(95 \% \mathrm{Cl})$} & $\beta$ (B; SE) \\
\hline Family affluence (ref. low/medium) & $0.90(0.70 ; 1.15)$ & $0.97(0.77 ; 1.23)$ & $0.95(0.78 ; 1.17)$ & $0.09(0.25 ; 0.09)^{* *}$ \\
\hline \multirow[t]{2}{*}{$\begin{array}{l}\text { Full model results (direct effects, all } \\
\text { background variables) }\end{array}$} & $\begin{array}{l}B / C=3927.32 \\
C A I C=3933.32\end{array}$ & $\begin{array}{l}B I C=3906.11 \\
C A I C=3912.11\end{array}$ & $\begin{array}{l}B / C=4674.49 \\
C A I C=4680.49\end{array}$ & $F(4,751)=10.54, p<.001$, adj. $R^{2}=0.05$ \\
\hline & \multicolumn{3}{|l|}{$\operatorname{Exp}(B),(95 \% \mathrm{Cl})$} & $\beta(B ; S E)$ \\
\hline Age & $1.07(1.01 ; 1.15)^{*}$ & $1.05(0.99 ; 1.12)^{\circ}$ & $1.04(0.99 ; 1.10)$ & $0.00(0.00 ; 0.02)$ \\
\hline Gender (ref. girls) & $0.63(0.51 ; 0.78) * * *$ & $\underset{* * *}{0.65}(0.53 ; 0.79)$ & $0.69(0.57 ; 0.82)^{* * *}$ & $0.21(0.46 ; 0.08)^{* * *}$ \\
\hline Family affluence (ref. low/medium) & $0.90(0.67 ; 1.19)$ & $0.91(0.70 ; 1.19)$ & $0.93(0.73 ; 1.18)$ & $0.09(0.26 ; 0.10)^{* *}$ \\
\hline BMI & $1.01(0.98 ; 1.05)$ & $1.01(0.98 ; 1.04)$ & $1.02(0.99 ; 1.05)$ & $-0.04(-0.01 ; 0.01)$ \\
\hline \multirow[t]{2}{*}{ Full model results (direct effects) } & $\begin{array}{l}B I C=3766.55 \\
C A I C=3775.55\end{array}$ & $\begin{array}{l}B / C=3718.42 \\
C A I C=3727.42\end{array}$ & $\begin{array}{l}B / C=4466.80 \\
C A I C=4475.80\end{array}$ & $F(7,716)=7.84 ; p<.001$, adj. $R^{2}=0.06$ \\
\hline & \multicolumn{3}{|l|}{$\operatorname{Exp}(B),(95 \% \mathrm{Cl})$} & $\beta$ (B; SE) \\
\hline Age & $0.99(0.92 ; 1.07)$ & $0.98(0.91 ; 1.04)$ & $0.98(0.92 ; 1.04)$ & $0.05(0.03 ; 0.03)$ \\
\hline Gender & $0.63(0.51 ; 0.79) * * *$ & $0.63(0.52 ; 0.77) * * *$ & $0.66(0.55 ; 0.79) * * *$ & $0.22(0.48 ; 0.08)^{* * *}$ \\
\hline Family Affluence & $0.92(0.68 ; 1.22)$ & $0.93(0.71 ; 1.23)$ & $0.94(0.74 ; 1.20)$ & $0.10(0.28 ; 0.09)^{* *}$ \\
\hline BMl & $1.00(0.97 ; 1.04)$ & $1.01(0.98 ; 1.04)$ & $1.01(0.98 ; 1.04)$ & $-0.03(-0.01 ; 0.01)$ \\
\hline Alcohol consumption & $1.02(0.98 ; 1.06)$ & $1.02(0.99 ; 1.06)$ & $1.03(1.00 ; 1.06)$ & $-0.05(-0.01 ; 0.01)$ \\
\hline Smoking & $1.11(0.98 ; 1.25)^{\circ}$ & $1.16(1.03 ; 1.30)^{*}$ & $1.12(1.01 ; 1.25)^{*}$ & $-0.02(-0.02 ; 0.04)$ \\
\hline Perceived sleep duration & $0.87(0.80 ; 0.94) * * *$ & $0.87(0.81 ; 0.94) * * *$ & $0.91(0.85 ; 0.98)^{* *}$ & $0.12(0.01 ; 0.03)^{* *}$ \\
\hline \multirow[t]{2}{*}{ Full model results (Interaction effects) } & $\begin{array}{l}B I C=3783.63 \\
C A I C=3795.63\end{array}$ & $\begin{array}{l}B I C=3735.21 \\
C A I C=3747.21\end{array}$ & $\begin{array}{l}B / C=4484.57 \\
C A I C=4496.57\end{array}$ & $F(10,713)=5.83, p<.001$, adj. $R^{2}=0.06$ \\
\hline & \multicolumn{3}{|l|}{$\operatorname{Exp}(B),(95 \% \mathrm{Cl})$} & $\beta$ (B; SE) \\
\hline Age & $0.99(0.92 ; 1.06)$ & $0.98(0.91 ; 1.04)$ & $0.98(0.92 ; 1.04)$ & $0.05(0.03 ; 0.03)$ \\
\hline Gender (ref. girls) & $0.63(0.51 ; 0.79)^{* * *}$ & $0.63(0.52 ; 0.78)^{* * *}$ & $0.67(0.56 ; 0.80)^{* * *}$ & $0.22(0.47 ; 0.08)^{* * *}$ \\
\hline Family Affluence (ref. low/medium) & $0.89(0.65 ; 1.20)$ & $0.90(0.68 ; 1.20)$ & $0.93(0.73 ; 1.20)$ & $0.09(0.26 ; 0.11)^{*}$ \\
\hline BMI & $1.00(0.97 ; 1.04)$ & $1.01(0.98 ; 1.04)$ & $1.01(0.98 ; 1.04)$ & $-0.03(-0.01 ; 0.01)$ \\
\hline Alcohol consumption & $1.12(0.98 ; 1.25)^{\circ}$ & $1.09(0.99 ; 1.21)^{\circ}$ & $1.07(0.98 ; 1.16)$ & $-0.13(-0.01 ; 0.04)$ \\
\hline Smoking & $1.02(0.77 ; 1.37)$ & $1.09(0.82 ; 1.44)$ & $1.15(0.90 ; 1.45)$ & $-0.11(-0.12 ; 0.10)$ \\
\hline Perceived sleep duration & $0.80(0.64 ; 0.99)^{*}$ & $0.82(0.70 ; 0.98)^{*}$ & $0.90(0.77 ; 1.05)$ & $-0.00(-0.00 ; 0.06)$ \\
\hline Alcohol * FAS & $0.92(0.81 ; 1.04)$ & $0.92(0.83 ; 1.03)$ & $0.96(0.87 ; 1.05)$ & $-0.02(-0.01 ; 0.04)$ \\
\hline Smoking * FAS & $1.08(0.79 ; 1.48)$ & $1.06(0.78 ; 1.44)$ & $0.96(0.74 ; 1.25)$ & $0.10(0.12 ; 0.11)$ \\
\hline Sleep * FAS & $1.09(0.87 ; 1.38)$ & $1.06(0.87 ; 1.29)$ & $1.01(0.85 ; 1.20)$ & $0.14(0.12 ; 0.07)$ \\
\hline Parsimonious model results (direct effects) & $\begin{array}{l}B I C=4753.20 \\
C A I C=4758.20\end{array}$ & $\begin{array}{l}B I C=4721.28 \\
C A I C=4726.28\end{array}$ & $\begin{array}{l}B / C=5694.01 \\
C A I C=5699.01\end{array}$ & $F(3,938)=21.26, p<.001$, adj. $R^{2}=0.06$ \\
\hline
\end{tabular}


Table 4 Regression analysis on the moderating role of family affluence in the relation between addictive behaviors and sleep, and mental health outcomes (Continued)

\begin{tabular}{|c|c|c|c|c|}
\hline & \multicolumn{4}{|c|}{ Dependent variable: mental health outcomes } \\
\hline & Depression $^{a}$ & Anxiety $^{a}$ & Stress $^{a}$ & Self-esteem $^{\mathbf{b}}$ \\
\hline & $\operatorname{Exp}(B),(95 \% \mathrm{Cl})$ & & & $\beta$ (B; SE) \\
\hline Gender (ref. girls) & $0.68(0.56 ; 0.82) * * *$ & $0.66(0.55 ; 0.79) * * *$ & $0.72(0.61 ; 0.84)^{* * *}$ & $0.19(0.41 ; 0.07)^{* * *}$ \\
\hline Family Affluence & Not included & Not included & Not included & $0.10(0.29 ; 0.09)^{* * *}$ \\
\hline Smoking & $1.15(1.05 ; 1.27)^{* *}$ & $1.17(1.07 ; 1.28) * * *$ & $1.16(1.07 ; 1.26)^{* * *}$ & Not included \\
\hline Sleep & $0.86(0.80 ; 0.92) * * *$ & $0.87(0.82 ; 0.93) * * *$ & $0.91(0.86 ; 0.97)^{* *}$ & $0.15(0.12 ; 0.02)^{* * *}$ \\
\hline
\end{tabular}

${ }^{\circ} p \leq .1 ;{ }^{*} p \leq 0.05 ;{ }^{* *} p \leq .01 ;{ }^{* * *} p \leq .001$

${ }^{a}$ Gamma generalized linear model

${ }^{\mathrm{b}}$ General linear model

Table 5 Regression analysis on the moderating role of family affluence in the relation between energy-balance related behaviors and mental health outcomes

\begin{tabular}{|c|c|c|c|c|}
\hline & \multicolumn{4}{|c|}{ Dependent variable: mental health outcomes } \\
\hline & Depression $^{\mathrm{a}}$ & Anxiety $^{a}$ & Stress $^{\mathrm{a}}$ & Self-esteem ${ }^{\mathbf{b}}$ \\
\hline \multirow[t]{2}{*}{ Full model results (direct effects) } & $\begin{array}{l}B I C=4559.69 \\
C A I C=4566.69\end{array}$ & $\begin{array}{l}B I C=4554.66 \\
C A I C=4561.66\end{array}$ & $\begin{array}{l}B I C=5457.89 \\
C A I C=5464.89\end{array}$ & $F(5,885)=11.80, p<.001$, adj. $R^{2}=0.06$ \\
\hline & \multicolumn{3}{|l|}{$\operatorname{Exp}(B),(95 \% \mathrm{Cl})$} & $\beta(B ; S E)$ \\
\hline Age & $1.06(1.00 ; 1.12)^{*}$ & $1.05(1.00 ; 1.11)^{\circ}$ & $1.05(1.00 ; 1.10)^{\circ}$ & $0.01(0.00 ; 0.02)$ \\
\hline Gender & $0.71(0.58 ; 0.86)^{* * *}$ & $0.69(0.57 ; 0.83)^{* * *}$ & $0.73(0.62 ; 0.86)^{* * *}$ & $0.18(0.39 ; 0.07)^{* * *}$ \\
\hline Family Affluence & $0.98(0.75 ; 1.26)$ & $1.01(0.80 ; 1.29)$ & $0.98(0.78 ; 1.21)$ & $0.07(0.20 ; 0.09)^{*}$ \\
\hline Days of breakfast & $0.95(0.91 ; 0.99)^{*}$ & $0.93(0.89 ; 0.96)^{* * *}$ & $0.94(0.91 ; 0.98)^{* * *}$ & $0.14(0.07 ; 0.02)^{* * * *}$ \\
\hline Physical activity days & $0.92(0.88 ; 0.97)^{* * *}$ & $0.96(0.92 ; 1.01)^{\circ}$ & $1.00(0.96 ; 1.04)$ & $0.04(0.02 ; 0.02)$ \\
\hline \multirow[t]{2}{*}{ Full model results (interaction effects) } & $\begin{array}{l}B / C=4572.84 \\
C A I C=4581.84\end{array}$ & $\begin{array}{l}B I C=4566.69 \\
C A I C=4575.69\end{array}$ & $\begin{array}{l}B I C=5469.15 \\
C A I C=5478.15\end{array}$ & $F(7,883)=8.70, p<.001$, adj. $R^{2}=0.06$ \\
\hline & \multicolumn{3}{|l|}{$\operatorname{Exp}(B),(95 \% \mathrm{Cl})$} & $\beta$ (B; SE) \\
\hline Age & $1.06(1.00 ; 1.12)^{*}$ & $1.05(1.00 ; 1.12)^{\circ}$ & $1.05(1.00 ; 1.10)^{\circ}$ & $0.01(0.00 ; 0.02)$ \\
\hline Gender (ref. girls) & $0.70(0.58 ; 0.86)^{* * *}$ & $0.69(0.58 ; 0.83)^{* * *}$ & $0.73(0.62 ; 0.86)^{* * *}$ & $0.18(0.39 ; 0.07)^{* * * *}$ \\
\hline Family Affluence & $0.96(0.74 ; 1.25)$ & $1.05(0.82 ; 1.35)$ & $1.01(0.81 ; 1.26)$ & $0.07(0.20 ; 0.10)^{*}$ \\
\hline Days of breakfast & $0.97(0.86 ; 1.09)$ & $0.93(0.85 ; 1.03)$ & $0.96(0.88 ; 1.05)$ & $0.06(0.03 ; 0.04)$ \\
\hline Physical activity days & $0.93(0.82 ; 1.06)$ & $0.89(0.79 ; 1.01)^{\circ}$ & $0.92(0.82 ; 1.03)$ & $0.11(0.06 ; 0.04)$ \\
\hline Days of breakfast * FAS & $0.96(0.86 ; 1.09)$ & $0.99(0.89 ; 1.11)$ & $0.98(0.89 ; 1.08)$ & $0.09(0.05 ; 0.04)$ \\
\hline Physical activity days * FAS & $0.99(0.86 ; 1.13)$ & $1.09(0.96 ; 1.25)$ & $1.10(0.98 ; 1.24)$ & $-0.08(-0.05 ; 0.05)$ \\
\hline \multirow[t]{2}{*}{ Parsimonious model results (direct effects) } & $\begin{array}{l}B I C=4619.84 \\
C A I C=4625.84\end{array}$ & $\begin{array}{l}B I C=4605.30 \\
C A I C=4611.30\end{array}$ & $\begin{array}{l}B I C=5574.12 \\
C A I C=5579.12\end{array}$ & $F(3,951)=20.12, p<.001$, adj. $R^{2}=0.06$ \\
\hline & \multicolumn{3}{|l|}{$\operatorname{Exp}(B),(95 \% \mathrm{Cl})$} & $\beta(B ; S E)$ \\
\hline Age & $1.05(1.00 ; 1.11)^{\circ}$ & $1.04(0.99 ; 1.10)$ & $1.04(1.00 ; 1.09)^{\circ}$ & Not included \\
\hline Gender (ref. girls) & $0.69(0.57 ; 0.85)^{* * *}$ & $0.68(0.56 ; 0.81)^{* * *}$ & $0.72(0.61 ; 0.85)^{* * *}$ & $0.18(0.39 ; 0.07)^{* * *}$ \\
\hline Family Affluence & Not included & Not included & Not included & $0.08(0.22 ; 0.09)^{*}$ \\
\hline Physical activity & $0.92(0.88 ; 0.97)^{* * *}$ & $0.96(0.92 ; 1.01)^{\circ}$ & Not included & Not included \\
\hline Days of breakfast & $0.95(0.91 ; 0.99)^{*}$ & $0.93(0.89 ; 0.97)^{* * *}$ & $0.94(0.91 ; 0.98)^{* *}$ & $0.15(0.07 ; 0.02)^{* * *}$ \\
\hline
\end{tabular}

${ }^{\circ} p \leq .1 ;{ }^{*} p \leq 0.05 ;{ }^{* *} p \leq .01 ;{ }^{* * *} p \leq .001$

${ }^{a}$ Gamma generalized linear model

${ }^{\mathrm{b}}$ General linear 


\section{Abbreviations}

SES: Socioeconomic status; FAS: Family affluence scale

\section{Acknowledgements}

Not applicable.

\section{Authors' contributions}

LM designed the research question, literature review, and was the main author in writing the manuscript. AS designed the study, collected the data, analyzed and interpreted data. GC1, GC2 and CP contributed in refining research questions and interpretation of findings. SC has assisted the authors with the statistical analysis during revision and was also involved in writing of the revised paper. All authors read and approved the final manuscript.

\section{Funding}

This work was supported by the Flemish agency for Innovation by Science and Technology (grant number 110051, 2012-2016). Ann DeSmet was supported by a Research Foundation Flanders fellowship (grant number FWO16/PDO/060, 12H6717N, 2016-2019). Carmen Peuters is supported by a grant from the Flemish Agency for Care and Health. The funding agencies had no role in any part of the study.

\section{Availability of data and materials}

The datasets used and/or analyzed during the current study are available from the corresponding author on reasonable request.

\section{Ethics approval and consent to participate}

The study received approval from the Ethics Committee of the Ghent University Hospital (2012/307, B670201214183). Adolescents provided written informed consent, parents provided passive informed consent. Parents were informed about the study through the school and received a telephone number and e-mail address from the researchers, via which they could notify that they did not want their child to participate. They were informed that when they did not contact the researchers, they agreed with participation of their child. The institutional review board approved the use of passive parental consent.

\section{Consent for publication}

Not applicable.

\section{Competing interests}

Ann DeSmet, co-author of this manuscript, is a member of the editorial board (Associate Editor) of BMC Public Health.

\section{Author details \\ ${ }^{1}$ Department of Movement and Sports Sciences, Faculty of Medicine and Health Sciences, Ghent University, Ghent, Belgium. ${ }^{2}$ Department of Experimental-Clinical and Health Psychology, Faculty of Psychology and Educational Sciences, Ghent University, Ghent, Belgium. ${ }^{3}$ Faculty of Psychology and Educational Sciences, Université Libre de Bruxelles, Brussels, Belgium. ${ }^{4}$ Department of Communication Studies, Faculty of Social Sciences, University of Antwerp, Antwerp, Belgium.}

\section{Received: 28 October 2019 Accepted: 12 June 2020}

Published online: 18 June 2020

\section{References}

1. WHO. Mental health: strengthening our response. https://www.who.int/ news-room/fact-sheets/detail/mental-health-strengthening-our-response. Accessed 18 Oct 2019

2. Gisle L. Geestelijke gezondheid. In: Van der Heyden J, Charrafeddine R, editors. Gezondheidsenquête 2013 Rapport 1: Gezondheid en Welzijn. Brussel: WIV-ISP; 2013. p. 796-954.

3. Kessler RC, Berglund P, Demler O, Jin R, Merikangas KR, Walters EE. Lifetime prevalence and age-of-onset distributions of DSM-IV disorders in the National Comorbidity Survey Replication. Arch Gen Psychiatry. 2005;62(6): 593-602.

4. WHO. Adolescent mental health. https://www.who.int/news-room/factsheets/detail/adolescent-mental-health. Accessed 12 Mar 2020.
5. Gisle L, Drieskens, S, Demarest, S, Van der Heyden, J. Geestelijke gezondheid gezondheidsenquête 2018. https://his.wiv-isp.be/nl/Gedeelde\%20\%2 Odocumenten/MH_NL_2018.pdf. Accessed 26 Mar 2020.

6. Cairns KE, Yap MBH, Pilkington PD, Jorm AF. Risk and protective factors for depression that adolescents can modify: a systematic review and metaanalysis of longitudinal studies. J Affect Disord. 2014;169:61-75.

7. Andrews G, Issakidis C, Sanderson K, Corry J, Lapsley H. Utilising survey data to inform public policy: comparison of the cost-effectiveness of treatment of ten mental disorders. Br J Psychiatry J Ment Sci. 2004;184(6):526-33.

8. Rickwood DJ, Deane FP, Wilson CJ. When and how do young people seek professional help for mental health problems? Med J Aust. 2007;187(7):S35

9. Ekkekakis P. Routledge handbook of physical activity and mental health. Abingdon: Routledge; 2013.

10. Velten J, Lavallee KL, Scholten S, Meyer AH, Zhang XC, Schneider S, et al. Lifestyle choices and mental health: a representative population survey. BMC Psychol. 2014;2(1):58.

11. Walsh R. Lifestyle and mental health. Am Psychol. 2011;66(7):579-92.

12. Rodelli M, De Bourdeaudhuij I, Dumon E, Portzky G, DeSmet A. Which healthy lifestyle factors are associated with a lower risk of suicidal ideation among adolescents faced with cyberbullying? Prev Med. 2018;113:32-40.

13. Kaneita Y, Ohida T, Osaki Y, Tanihata T, Minowa M, Suzuki K, et al. Association between mental health status and sleep status among adolescents in Japan: a nationwide cross-sectional survey. J Clin Psychiatry. 2007;68(9):1426-35.

14. Calfas KJ, Taylor WC. Effects of physical activity on psychological variables in adolescents. Pediatr Exerc Sci. 1994;6(4):406-23.

15. Biddle SJ, Asare M. Physical activity and mental health in children and adolescents: a review of reviews. Br J Sports Med. 2011:45(11):886-95.

16. Rodriguez-Ayllon M, Cadenas-Sanchez C, Estevez-Lopez F, Munoz NE, MoraGonzalez J, Migueles JH, et al. Role of physical activity and sedentary behavior in the mental health of preschoolers, children and adolescents: a systematic review and meta-analysis. Sports Med. 2019;49(9):1383-410.

17. Hamilton NA, Nelson CA, Stevens N, Kitzman H. Sleep and psychological well-being. Soc Indic Res. 2007:82(1):147-63.

18. Tremblay MS, Inman JW, Willms JD. The relationship between physical activity, self-esteem, and academic achievement in 12-year-old children. Pediatr Exerc Sci. 2000;12(3):312-23.

19. Ekeland $E_{1}$ Heian $F$, Hagen KB. Can exercise improve self esteem in children and young people? A systematic review of randomised controlled trials. $\mathrm{Br} J$ Sports Med. 2005;39(11):792-8.

20. McGee R, Williams S. Does low self-esteem predict health compromising behaviours among adolescents? J Adolesc. 2000;23(5):569-82.

21. Kinnunen T, Haukkala A, Korhonen T, Quiles ZN, Spiro A 3rd, Garvey AJ. Depression and smoking across 25 years of the normative aging study. Int J Psychiatry Med. 2006;36(4):413-26.

22. Abernathy $T J$, Massad $L$, Romanodwyer $L$. The relationship between smoking and self-esteem. Adolescence. 1995;30(120):899-907.

23. Chaiton MO, Cohen JE, O'Loughlin J, Rehm J. A systematic review of longitudinal studies on the association between depression and smoking in adolescents. BMC Public Health. 2009;9(1):356.

24. Wild LG, Flisher AJ, Bhana A, Lombard C. Associations among adolescent risk behaviours and self-esteem in six domains. J Child Psychol Psychiatry. 2004:45(8):1454-67.

25. Scheier LM, Botvin GJ, Griffin KW, Diaz T. Dynamic growth models of selfesteem and adolescent alcohol use. J Early Adolesc. 2000;20(2):178-209.

26. Lazzeri G, Ahluwalia N, Niclasen B, Pammolli A, Vereecken C, Rasmussen M, et al. Trends from 2002 to 2010 in daily breakfast consumption and its socio-demographic correlates in adolescents across 31 countries participating in the HBSC study. PLoS One. 2016;11(3):e0151052.

27. Guthold R, Stevens GA, Riley LM, Bull FC. Global trends in insufficient physical activity among adolescents: a pooled analysis of 298 populationbased surveys with 1.6 million participants. Lancet Child Adolesc. 2020;4(1): 23-35.

28. Delaruelle K, Dierckens M, Vandendriessche A, Deforche B. Studie Jongeren en Gezondheid, Deel 3: gezondheid en welzijn - Uitgelicht: slaap [Factsheet]. http://www.jongeren-en-gezondheid.ugent.be/materialen/ factsheets-vlaanderen/gezondheid-en-welzijn/. Accessed 23 Mar 2020.

29. Dierckens M, De Clercq B, Deforche B. Studie Jongeren en Gezondheid, Deel 4: gezondheidsgedrag - Beweging en sedentair gedrag [Factsheet]. http://www.jongeren-en-gezondheid.ugent.be/materialen/factsheetsvlaanderen/fysieke-activiteit-en-vrije-tijd/. Accessed 23 Mar 2020. 
30. Dierckens M, De Clercq B, Deforche B. Studie Jongeren en Gezondheid, Deel 4: gezondheidsgedrag - Voeding [Factsheet]. http://www.jongeren-engezondheid.ugent.be/materialen/factsheets-vlaanderen/voeding/. Accessed 23 Mar 2020.

31. Inchley JC, Currie DB, Vieno A, Torsheim T, Ferreira-Borges C, Weber M, et al. Adolescent alcohol-related behaviours: Trends and inequalities in the WHO European Region, 2002-2014: WHO Regional Office for Europe. 2018.

32. Dierckens M, De Clercq B, Deforche B. Studie Jongeren en Gezondheid, Deel 4: gezondheidsgedrag - Risicogedragingen: alcohol, roken en drugs [Factsheet]. http://www.jongeren-en-gezondheid.ugent.be/materialen/ factsheets-vlaanderen/alcohol-roken-en-andere-drugs/. Accessed $23 \mathrm{Mar}$ 2020.

33. Mackenbach JP, Stirbu I, Roskam AJ, Schaap MM, Menvielle G, Leinsalu M, et al. Socioeconomic inequalities in health in 22 European countries. N Engl J Med. 2008;358(23):2468-81.

34. Richter M, Vereecken CA, Boyce W, Maes L, Gabhainn SN, Currie CE. Parental occupation, family affluence and adolescent health behaviour in 28 countries. Int J Public Health. 2009;54(4):203-12.

35. Elgar FJ, Pförtner T, Moor I, De Clercq B, Stevens GW, Currie C. Socioeconomic inequalities in adolescent health 2002-2010: a time-series analysis of 34 countries participating in the health behaviour in school-aged children study. Lancet. 2015;385(9982):2088-95.

36. van Lenthe FJ, de Bourdeaudhuij I, Klepp KI, Lien N, Moore L, Faggiano F, et al. Preventing socioeconomic inequalities in health behaviour in adolescents in Europe: background, design and methods of project TEENAGE. BMC Public Health. 2009;9(1):125.

37. Zambon A, Boyce W, Cois E, Currie C, Lemma P, Dalmasso P, et al. Do welfare regimes mediate the effect of socioeconomic position on health in adolescence? A cross-national comparison in Europe, North America, and Israel. Int J Health Serv. 2006;36(2):309-29.

38. Reiss F. Socioeconomic inequalities and mental health problems in children and adolescents: a systematic review. Soc Sci Med. 2013;90:24-31.

39. Robins RW, Trzesniewski KH, Tracy JL, Gosling SD, Potter J. Global selfesteem across the life span. Psychol Aging. 2002;17(3):423-34.

40. Twenge JM, Campbell WK. Self-esteem and socioeconomic status: a metaanalytic review. Personal Soc Psychol Rev. 2002;6(1):59-71.

41. Marco CA, Wolfson AR, Sparling M, Azuaje A. Family socioeconomic status and sleep patterns of young adolescents. Behav Sleep Med. 2012;10(1):70-80.

42. Keski-Rahkonen A, Kaprio J, Rissanen A, Virkkunen M, Rose RJ. Breakfast skipping and health-compromising behaviors in adolescents and adults. Eur J Clin Nutr. 2003;57(7):842-53.

43. Moore PJ, Adler NE, Williams DR, Jackson JS. Socioeconomic status and health: the role of sleep. Psychosom Med. 2002;64(2):337-44.

44. Tuinstra J, Groothoff JW, Van Den Heuvel WJ, Post D. Socio-economic differences in health risk behavior in adolescence: do they exist? Soc Sci Med. 1998;47(1):67-74

45. Felden EP, Leite CR, Rebelatto CF, Andrade RD, Beltrame TS. Sleep in adolescents of different socioeconomic status: a systematic review. Rev Paul Pediatr. 2015;33(4):467-73.

46. Melotti R, Heron J, Hickman M, Macleod J, Araya R, Lewis G. Adolescent alcohol and tobacco use and early socioeconomic position: the ALSPAC birth cohort. Pediatrics. 2011;127(4):e948-e55.

47. Hanson MD, Chen E. Socioeconomic status and health behaviors in adolescence: a review of the literature. J Behav Med. 2007;30(3):263-85.

48. Finkelstein DM, Kubzansky LD, Goodman E. Social status, stress, and adolescent smoking. J Adolesc Health. 2006;39(5):678-85.

49. Goodman E, Huang B. Socioeconomic status, depressive symptoms, and adolescent substance use. Arch Pediatr Adolesc Med. 2002;156(5):448-53.

50. Vereecken C, Dupuy M, Rasmussen M, Kelly C, Nansel TR, Al Sabbah H, et al. Breakfast consumption and its socio-demographic and lifestyle correlates in schoolchildren in 41 countries participating in the HBSC study. Int J Public Health. 2009;54(2):180-90.

51. Humensky JL. Are adolescents with high socioeconomic status more likely to engage in alcohol and illicit drug use in early adulthood? Subst Abuse Treat Prev Policy. 2010;5(1):1.

52. Lemstra M, Bennett NR, Neudorf C, Kunst A, Nannapaneni U, Warren LM et al. A meta-analysis of marijuana and alcohol use by socio-economic status in adolescents aged 10-15 years. Can J Public Health. 2008;99(3):172-7.

53. Richter M, Kuntsche E, de Looze M, Pförtner T-K. Trends in socioeconomic inequalities in adolescent alcohol use in Germany between 1994 and 2006. Int J Public Health. 2013;58(5):777-84.
54. Motl RW, Birnbaum AS, Kubik MY, Dishman RK. Naturally occurring changes in physical activity are inversely related to depressive symptoms during early adolescence. Psychosom Med. 2004;66(3):336-42.

55. Boyce W, Torsheim T, Currie C, Zambon A. The family affluence scale as a measure of national wealth: validation of an adolescent self-report measure. Soc Indic Res. 2006;78(3):473-87.

56. Currie C, Inchley J, Molcho M, Lenzi M, Veselska Z, Wild F. Health behaviour in school-aged children protocol: background, methodology and mandatory items for the 2013/2014 survey. Child \& Adolescent Health Research Unit; 2014.

57. Inchley J, Currie D. Growing up unequal: gender and socioeconomic differences in young people's health and well-being. Health Behav Sch Aged Child. 2013;2014:2-3.

58. French SA, Perry CL, Leon GR, Fulkerson JA. Self-esteem and change in body mass index over 3 years in a cohort of adolescents. Obes Res. 1996; 4(1):27-33.

59. Schnohr CW, Kreiner S, Due EP, Currie C, Boyce W, Diderichsen F. Differential item functioning of a family affluence scale: validation study on data from HBSC 2001/02. Soc Indic Res. 2008;89(1):79-95.

60. Currie C, Molcho M, Boyce W, Holstein B, Torsheim T, Richter M. Researching health inequalities in adolescents: the development of the Health Behaviour in School-Aged Children (HBSC) family affluence scale. Soc Sci Med. 2008; 66(6):1429-36

61. Molcho M, Gabhainn SN, Kelleher CC. Assessing the use of the Family Affluence Scale (FAS) among Irish schoolchildren. Ir Med J. 2007;100(8):37-9.

62. Hobza V, Hamrik Z, Bucksch J, De Clercq B. The family affluence scale as an Indicator for socioeconomic status: validation on regional income differences in the Czech Republic. Int J Environ Res Public Health. 2017 ; 14(12):1540

63. Kehoe $\mathrm{S}, \mathrm{O}$ 'Hare $\mathrm{L}$. The reliability and validity of the family affluence scale. Eff Educ. 2010;2(2):155-64.

64. Pearson N, Biddle SJ, Gorely T. Family correlates of breakfast consumption among children and adolescents. A systematic review. Appetite. 2009;52(1):1-7.

65. Wong CL, Mullan BA. Predicting breakfast consumption: an application of the theory of planned behaviour and the investigation of past behaviour and executive function. Br J Health Psychol. 2009;14(Pt 3):489-504.

66. Post A, Gilljam H, Rosendahl I, Meurling L, Bremberg S, Galanti MR. Validity of self reports in a cohort of Swedish adolescent smokers and smokeless tobacco (snus) users. Tob Control. 2005;14(2):114-7.

67. HBSC. Health Behaviour in School-Aged Children. http://www.hbsc.org/. Accessed 25 Mar 2020

68. Currie C, Inchley J, Molcho M, Lenzi M, Veselska Z, Wild F. Health behaviour in school-aged children (HBSC) study protocol: background, methodology and mandatory items for the 2013/14 survey; 2014.

69. Griebler R, Molcho M, Samdal O, Inchley J, Dür W, Currie C. Health behaviour in school-aged children: a World Health Organization crossnational study. Research protocol for the 2009/2010 survey. Vienna: LBIHPR and Edinburgh: CAHRU; 2010.

70. Liu Y, Wang M, Tynjala J, LV Y, Villberg J, Zhang Z, et al. Test-retest reliability of selected items of Health Behaviour in School-aged Children (HBSC) survey questionnaire in Beijing, China. BMC Med Res Methodol. 2010;10(1):73.

71. Nascimento-Ferreira MV, Collese TS, de Moraes ACF, Rendo-Urteaga T, Moreno LA, Carvalho HB. Validity and reliability of sleep time questionnaires in children and adolescents: a systematic review and meta-analysis. Sleep Med Rev. 2016:30:85-96.

72. Shea TL, Tennant A, Pallant JF. Rasch model analysis of the Depression, Anxiety and Stress Scales (DASS). BMC Psychiatry. 2009;9(1):21.

73. Szabó M. The short version of the Depression Anxiety Stress Scales (DASS21): Factor structure in a young adolescent sample. J Adolesc. 2010;33(1):8

74. Mann M, Hosman CM, Schaalma HP, de Vries NK. Self-esteem in a broadspectrum approach for mental health promotion. Health Educ Res. 2004; 19(4):357-72.

75. Robins RW, Hendin HM, Trzesniewski KH. Measuring global self-esteem: construct validation of a single-item measure and the Rosenberg selfesteem scale. Pers Soc Psychol B. 2001;27(2):151-61.

76. Franck E, De Raedt R, Barbez C, Rosseel Y. Psychometric properties of the Dutch Rosenberg self-esteem scale. Psychol Belg. 2008;48(1):25-34.

77. Zimprich D, Perren S, Hornung R. A two-level confirmatory factor analysis of a modified Rosenberg self-esteem scale. Educ Psychol Meas. 2005;65(3):465-81.

78. George D. SPSS for windows step by step: a simple study guide and reference, 17.0 update, 10/e: Pearson Education India; 2011. 
79. Ng VK, Cribbie RA. Using the gamma generalized linear model for modeling continuous, skewed and heteroscedastic outcomes in psychology. Curr Psychol. 2017;36(2):225-35.

80. Field A. Discovering statistics using IBM SPSS statistics, 4th edition Carmichael M, editor. London: SAGE Publications; 2014. p. 916.

81. Bellis MA, Hughes $K$, Morleo M, Tocque K, Hughes S, Allen T, et al. Predictors of risky alcohol consumption in schoolchildren and their implications for preventing alcohol-related harm. Subst Abuse Treat Prev Policy. 2007;2(1):15.

82. Richter $\mathrm{M}$, Leppin $\mathrm{A}$. Trends in socio-economic differences in tobacco smoking among German schoolchildren, 1994-2002. Eur J Pub Health. 2007; 17(6):565-71.

83. Myklestad I, Røysamb E, Tambs K. Risk and protective factors for psychological distress among adolescents: a family study in the NordTrøndelag health study. Soc Psychiatry Psychiatr Epidemiol. 2012;47(5):771-82.

84. Wight RG, Botticello AL, Aneshensel CS. Socioeconomic context, socia support, and adolescent mental health: a multilevel investigation. J Youth Adolesc. 2006;35(1):115-26.

85. Barry CT, Sidoti CL, Briggs SM, Reiter SR, Lindsey RA. Adolescent social media use and mental health from adolescent and parent perspectives. J Adolesc. 2017;61:1-11.

86. Bakker MP, Ormel J, Verhulst FC, Oldehinkel AJ. Peer stressors and gender differences in adolescents' mental health: the TRAlLS study. J Adolesc Health. 2010;46(5):444-50.

87. Elgar FJ, Roberts C, Tudor-Smith C, Moore L. Validity of self-reported height and weight and predictors of bias in adolescents. J Adolesc Health. 2005; 37(5):371-5.

88. Lassale C, Batty GD, Baghdadli A, Jacka F, Sánchez-Villegas A, Kivimäki M, et al. Healthy dietary indices and risk of depressive outcomes: a systematic review and meta-analysis of observational studies. Mol Psychiatry. 2019; 24(7):965-86.

89. Khalid S, Williams CM, Reynolds SA. Is there an association between diet and depression in children and adolescents? A systematic review. Br J Nutr. 2016;116(12):2097-108

90. Sanhueza C, Ryan L, Foxcroft DR. Diet and the risk of unipolar depression in adults: systematic review of cohort studies. J Hum Nutr Diet. 2013;26(1):56-70.

\section{Publisher's Note}

Springer Nature remains neutral with regard to jurisdictional claims in published maps and institutional affiliations.

Ready to submit your research? Choose BMC and benefit from:

- fast, convenient online submission

- thorough peer review by experienced researchers in your field

- rapid publication on acceptance

- support for research data, including large and complex data types

- gold Open Access which fosters wider collaboration and increased citations

- maximum visibility for your research: over $100 \mathrm{M}$ website views per year

At BMC, research is always in progress.

Learn more biomedcentral.com/submissions 